\title{
AVALIAÇÃO DO POTENCIAL DE COPRODUTOS DA CADEIA DO BIODIESEL PARA PRODUÇÃO DE ETANOL
}

\author{
A. L. MACEDO ${ }^{1}$, R. S. SANTOS ${ }^{1}$, R. K. LANGBHEN $^{3}$, R. G. C. $\operatorname{SILVA}^{3}$, A. A. SILVA ${ }^{1}$, H. T. \\ L. dos SANTOS $^{4}$, T. LAUAR $^{3}$, C. A. FERREIRA ${ }^{1}$, L. A. PANTOJA ${ }^{3}$, A. S. SANTOS ${ }^{2}$ \\ ${ }^{1}$ Universidade Federal dos Vales do Jequitinhonha e Mucuri, Programa de Pós Graduação em \\ Biocombustíveis \\ ${ }^{2}$ Universidade Federal dos Vales do Jequitinhonha e Mucuri, Departamento de Ciências Básicas \\ ${ }^{3}$ Universidade Federal dos Vales do Jequitinhonha e Mucuri, Instituto de Ciências e Tecnologia \\ ${ }^{4}$ Universidade de São Paulo, Departamento de Biotecnologia \\ E-mail para contato: alicelopesm@hotmail.com
}

\begin{abstract}
RESUMO - A produção de etanol a partir de resíduos de oleaginosas figura uma estratégia que pode complementar ou até suprir a demanda de álcool em reações de transesterificação nas indústrias de biodiesel. O presente estudo teve por objetivo avaliar a composição química e o potencial das tortas de algodão, girassol, pinhãomanso, mamona, tremoço, macaúba, corda-de-viola, pequi, dendê, crambe e nabo forrageiro para produção de etanol. A estimativa de produção por tonelada das tortas referidas variou de $100 \mathrm{~L} /$ ton a $395 \mathrm{~L} /$ ton. As projeções feitas a partir das biomassas supracitadas, com exceção da torta de mamona e de nabo forrageiro, demonstraram atender a demanda de álcool para produção de biodiesel. Observou-se grande potencial estimado de produtividade de etanol paras as tortas de algodão, dendê e macaúba, com valores de 520, 900 e $1000 \mathrm{~L} / \mathrm{ha}$, respectivamente. Assim, a produção de etanol a partir de tortas pode ser promissora para atender à cadeia produtiva do biodiesel.
\end{abstract}

\section{INTRODUÇÃO}

A matriz atual do consumo global de energia é dominada por combustíveis fósseis (petróleo, gás, carvão), fontes não-renováveis, cuja queima vem acompanhada das conhecidas consequências ambientais, sobretudo em razão das emissões de gases poluentes, como COx, SOx e NOx, para a atmosfera (Misra, 2013). Neste panorama, tem-se observado o crescimento de pesquisas voltadas para o desenvolvimento de energias alternativas. $\mathrm{O}$ biodiesel, em particular, teve crescimento significativo nos últimos oito anos (Brasil, 2013). Concomitante com o aumento da produção de biodiesel cresce também a geração de resíduos oriundos da sua cadeia produtiva, tais como a glicerina e as tortas, tornando necessário um enfoque especial na destinação destes subprodutos. A torta ou farelo, gerada na extração do óleo, possui potencial para agregar valor ao biodiesel, mas, para isso, suas potencialidades nutricionais e econômicas devem ser explanadas. Atualmente, os principais empregos das tortas são na adubação orgânica, alimentação animal e geração de energia por queima direta.

Em nível mundial, as indústrias de biodiesel são responsáveis por captar 10,2\% da produção de óleo vegetais anualmente (BiodieselBr.com). Algumas oleaginosas já são consolidadas na produção de matéria-prima para esse setor e outras estão sendo pesquisadas como produtoras em potencial de óleos. Dentre as oleaginosas já consolidadas, pode-se citar: o dendezeiro (Elaeis guineensis), algodoeiro (Gossypium ssp) e o girassol (Helianthus annuus). Já dentre as oleaginosas que estão sendo alvos de pesquisa, destaca-se a mamona (Ricinus communis L.), pinhão-manso (Jatropha curcas L.), pequi (Caryocar brasiliense 
Camb), tremoço (Lupinus albus), nabo forrageiro (Raphanus sativus L.), crambe (Crambe abyssinica) e a corda-de-viola (Ipomoea triloba L.)

Os resíduos gerados pela extração lipídica das oleaginosas citadas anteriormente são biomassas que apresentam significativo percentual de polissacarídeos (amido, celulose e hemicelulose), que poderiam ser aproveitados para a produção de etanol através de processos hidrolíticos e fermentativo. A produção de etanol a partir de resíduos figura como uma estratégia economicamente interessante para aplicação em reações de transesterificação, principal rota de produção de biodiesel (Math et al., 2010). Neste contexto, o presente estudo teve por objetivo avaliar a composição química e o potencial das tortas de algodão, girassol, pinhão-manso, mamona, tremoço, macaúba, corda-de-viola, pequi, dendê, crambe e nabo forrageiro, coprodutos das indústrias de biodiesel, para produção de etanol.

\section{METODOLOGIA}

\subsection{Obtenção das tortas}

As tortas foram obtidas por doação ou preparadas no Laboratório de Bioprocessos e Biotransformação da Universidade Federal dos Vales do Jequitinhonha e Mucuri- UFVJM. Todas as tortas utilizadas foram submetidas a um processo de extração lipídica com éter etílico, para eliminação de óleo residual. As tortas desengorduradas foram secas em estufa com circulação de ar forçada a $60{ }^{\circ} \mathrm{C}$, por 48 horas, para eliminação de umidade e solvente residual. Em seguida, foram cominuídas em moinho de facas e posteriormente peneiradas sobre malhas de 0,5 e $0,2 \mathrm{~mm}$.

\subsection{Caracterização química}

As tortas foram submetidas a análises de caracterização centesimal, na qual foram avaliados: umidade, cinzas, proteínas totais e lipídios totais (Association of Oficial Analytical Chemistry - AOAC 1992); fibra solúvel em detergente neutro (FDN), fibra solúvel em detergente ácido (FDA), celulose, hemicelulose e lignina (Van Soest, 1966, 1967); açúcares solúveis totais (Dubois et al., 1956) e amido (McCready et al., 1950).

\subsection{Estimativa da produção de etanol}

O potencial teórico para produção de etanol foi calculado considerando a completa transformação hidrolítica de amido e celulose em glicose e de hemicelulose em xilose e conversão fermentativa teórica de $1 \mathrm{~g}$ dos monossacarídeos em 0,511 g de etanol (Kosaric, 2001), conforme Equação 1. A projeção da produtividade de etanol em litros por hectare de plantio foi calculado de acordo com a Equação 2, utilizando-se as produtividades de cada oleaginosa descritas na Tabela 1.

Produção de etanol $\left(\right.$ L.ton $\left.^{-1}\right)=(\%$ polissacarídeo $\times 1000 \times 0,511) / 0,789$

\footnotetext{
Produtividade etanol $\left(\right.$ L.ha $\left.{ }^{-1}\right)=$ Etanol $\left(\right.$ L.ton $\left.^{-1}\right) \times$ produtividade torta $\left(\right.$ ton.ha $\left.{ }^{-1}\right)$
} 
Tabela 1 - Valores de produtividade de torta e quantidade de torta produzida por tonelada de óleo extraído baseados no percentual de óleo e produtividade da oleaginosa

\begin{tabular}{|c|c|c|c|c|c|c|}
\hline \multirow{2}{*}{ Oleaginosa } & \multirow{2}{*}{$\begin{array}{c}\% \\
\text { óleo }\end{array}$} & \multicolumn{3}{|c|}{ Produtividade (ton.ha ${ }^{-1}$ ) } & \multirow{2}{*}{$\begin{array}{l}\text { Ton. torta/ton. } \\
\text { óleo extraído }\end{array}$} & \multirow{2}{*}{ Referências } \\
\hline & & Fruto/semente & Óleo* & Torta* & & \\
\hline Polpa de Macaúba & 29 & 14 & 4,1 & 9,90 & 2,4 & $\begin{array}{l}\text { Tickell, 2000; } \\
\text { Coimbra, 2011 }\end{array}$ \\
\hline Mamona & 50 & 2,34 & 1,17 & 1,17 & 1,2 & $\begin{array}{c}\text { Ramanjaneyulu et } \\
\text { al., } 2013\end{array}$ \\
\hline Pinhão-manso & 39 & 3,9 & 1,5 & 2,4 & 1,6 & $\begin{array}{c}\text { Corzo-Valladares } \\
\text { et al., 2012; } \\
\text { Drumond et al., } \\
2010\end{array}$ \\
\hline Girassol & 38 & 1,6 & 0,52 & 0,98 & 1,9 & $\begin{array}{l}\text { Bergmann, 2013; } \\
\quad \text { Conab } 2013\end{array}$ \\
\hline Caroço de Algodão & 20 & 2,3 & 0,46 & 1,84 & 4,0 & $\begin{array}{l}\text { Bergmann, 2013; } \\
\text { Conab } 2013\end{array}$ \\
\hline Tremoço & 10 & 2,4 & 0,24 & 2,16 & 9,0 & $\begin{array}{c}\text { Ribeiro, } 2006 \\
\text { Bessa et al., } 2009\end{array}$ \\
\hline Pequi & 30 & 1,8 & 0,53 & 1,2 & 2,26 & $\begin{array}{l}\text { Macedo, } 2011 \\
\text { Vera, } 2007\end{array}$ \\
\hline Nabo forrageiro & 35 & 1,2 & 0,55 & 0,95 & 1,73 & $\begin{array}{l}\text { Dambiski, } 2007 \\
\text { Valle, } 2009\end{array}$ \\
\hline Crambe & 40 & 1,5 & 0,4 & 1,1 & 2,75 & Masetto, 2009 \\
\hline Dendê & 41 & 13 & 5,3 & 7,7 & 1,45 & $\begin{array}{l}\text { Silva, } 2006 \\
\text { Santos, } 2010\end{array}$ \\
\hline
\end{tabular}

\subsection{Avaliação da demanda de etanol nas indústrias de biodiesel}

A quantidade de etanol utilizada em processos de transesterificação depende do tipo de catalisador e fonte de triglicerídeos utilizados (Marchetti et al., 2007). Neste estudo, a relação estequiométrica molar mínina (3:1 álcool/óleo) foi generalizada de forma a determinar o volume aproximado de álcool necessário para a transesterificação de uma tonelada do trioleato de glicerina (triglicerídeo de ácido oléico), principal constituinte da maioria dos óleos vegetais. Assim, são necessários cerca de $200 \mathrm{~L}$ de álcool neste processo. O cálculo foi realizado conforme a Equação 3.

$$
\text { Etanol }(L)=\operatorname{Etanol}\left(\text { L.ton }^{-1}\right) \times \text { Torta }\left(\text { ton.ton de óleo }{ }^{-1}\right)
$$

\section{RESULTADOS E DISCUSSÃO}

As composições químicas das tortas estão apresentadas na Tabela 2 e a estimativa da produção em litros de etanol por tonelada de torta está expressa na Tabela 3. 
Tabela 2 - Composição química de tortas oriundas da cadeia do biodiesel.

\begin{tabular}{|c|c|c|c|c|c|c|c|c|c|c|c|}
\hline Parâmetros & Algodão & $\begin{array}{l}\text { Corda-de- } \\
\text { viola }\end{array}$ & Crambe & Dendê & Girassol & Macaúba & Mamona & $\begin{array}{c}\text { Nabo } \\
\text { forrageiro }\end{array}$ & Pequi & $\begin{array}{c}\text { Pinhão- } \\
\text { manso }\end{array}$ & Tremoço \\
\hline Umidade & $\begin{array}{c}3,27 \pm \\
0,08\end{array}$ & $8,35 \pm 0,17$ & $\begin{array}{c}6,82 \pm \\
0,15\end{array}$ & $\begin{array}{c}5,14 \pm \\
0,07\end{array}$ & $0,46 \pm 0,01$ & $7,80 \pm 0,23$ & $\begin{array}{c}3,49 \pm \\
0,18\end{array}$ & $6,97 \pm 0,27$ & $\begin{array}{c}3,87 \pm \\
0,19\end{array}$ & $2,18 \pm 0,17$ & $5,47 \pm 0,13$ \\
\hline Lipídeos & $\begin{array}{c}3,38 \pm \\
0,06\end{array}$ & $9,92 \pm 0,11$ & $\begin{array}{c}5,57 \pm \\
0,43\end{array}$ & $\begin{array}{c}2,72 \pm \\
0,08\end{array}$ & $0,03 \pm 0,01$ & $4,70 \pm 0,15$ & $\begin{array}{c}2,04 \pm \\
0,38\end{array}$ & $2,94 \pm 0,03$ & $\begin{array}{c}6,17 \pm \\
1,81\end{array}$ & $1,15 \pm 0,08$ & $0,49 \pm 0,01$ \\
\hline Cinzas & $\begin{array}{c}3,70 \pm \\
0,13\end{array}$ & $3,59 \pm 0,26$ & $\begin{array}{c}4,89 \pm \\
0,17\end{array}$ & $\begin{array}{c}4,68 \pm \\
0,57\end{array}$ & $5,62 \pm 0,03$ & $4,19 \pm 0,16$ & $\begin{array}{c}6,34 \pm \\
0,17\end{array}$ & $7,28 \pm 1,81$ & $\begin{array}{c}4,71 \pm \\
0,24\end{array}$ & $6,24 \pm 0,13$ & $3,02 \pm 0,09$ \\
\hline PB & $\begin{array}{c}23,04 \pm \\
0,05\end{array}$ & $\begin{array}{c}10,28 \pm \\
0,85\end{array}$ & $\begin{array}{c}35,68 \pm \\
0,08\end{array}$ & $\begin{array}{c}5,44 \pm \\
0,19\end{array}$ & $28,74 \pm 2,31$ & 8,15 & $\begin{array}{c}35,09 \pm \\
0,30\end{array}$ & $\begin{array}{c}47,65 \pm \\
1,35\end{array}$ & $\begin{array}{c}29,51 \pm \\
0,86\end{array}$ & $32,92 \pm 0,78$ & $39,54 \pm 0,1$ \\
\hline AST & $\begin{array}{c}4,44 \pm \\
0,55\end{array}$ & $1,47 \pm 0,17$ & $\begin{array}{c}5,10 \pm \\
0,39\end{array}$ & $\begin{array}{c}4,56 \pm \\
0,11\end{array}$ & $7,76 \pm 0,93$ & $11,48 \pm 0,62$ & $\begin{array}{c}4,25 \pm \\
0,07\end{array}$ & $9,61 \pm 0,68$ & $\begin{array}{c}40,75 \pm \\
4,45\end{array}$ & $5,29 \pm 0,15$ & $9,82 \pm 0,37$ \\
\hline Amido & $\begin{array}{c}2,39 \pm \\
0,35\end{array}$ & $8,67 \pm 0,72$ & $\begin{array}{c}2,18 \pm \\
0,62\end{array}$ & $\begin{array}{c}5,02 \pm \\
0,54\end{array}$ & $4,04 \pm 0,38$ & $23,16 \pm 0,95$ & $\begin{array}{c}5,74 \pm \\
0,22\end{array}$ & $3,58 \pm 0,20$ & $\begin{array}{c}27,14 \pm \\
0,86\end{array}$ & $4,97 \pm 0,33$ & $6,06 \pm 0,38$ \\
\hline FDA & $\begin{array}{c}45,32 \pm \\
1,80\end{array}$ & $\begin{array}{c}50,62 \pm \\
0,98\end{array}$ & $\begin{array}{c}32,54 \pm \\
1,27\end{array}$ & $\begin{array}{c}72,01 \pm \\
1,38\end{array}$ & $32,90 \pm 0,66$ & $14,12 \pm 0,91$ & $\begin{array}{c}46,22 \pm \\
0,87\end{array}$ & $\begin{array}{c}16,12 \pm \\
0,45\end{array}$ & $\begin{array}{c}25,91 \pm \\
0,58\end{array}$ & $51,9 \pm 2,10$ & $9,29 \pm 0,01$ \\
\hline FDN & $\begin{array}{c}52,19 \pm \\
0,14\end{array}$ & $\begin{array}{c}69,63 \pm \\
0,72\end{array}$ & $\begin{array}{c}50,93 \pm \\
2,91\end{array}$ & $\begin{array}{c}83,15 \pm \\
1,96\end{array}$ & $53,02 \pm 0,76$ & $23,72 \pm 0,36$ & $\begin{array}{c}52,28 \pm \\
1,01\end{array}$ & $\begin{array}{c}23,16 \pm \\
0,06\end{array}$ & $\begin{array}{c}42,16 \pm \\
0,73\end{array}$ & $62,67 \pm 3,13$ & $\begin{array}{c}21,14 \pm 0,6 \\
4\end{array}$ \\
\hline Celulose & $\begin{array}{c}33,29 \pm \\
1,13\end{array}$ & $\begin{array}{c}33,36 \pm \\
0,70\end{array}$ & $\begin{array}{c}18,92 \pm \\
0,68\end{array}$ & $\begin{array}{c}30,36 \pm \\
3,66\end{array}$ & $23,22 \pm 0,30$ & $11,49 \pm 1,08$ & $\begin{array}{c}12,59 \pm \\
0,96\end{array}$ & $9,17 \pm 0,67$ & $\begin{array}{c}16,64 \pm \\
0,46\end{array}$ & $17,46 \pm 0,26$ & $\begin{array}{c}10,10 \pm 0,0 \\
4\end{array}$ \\
\hline Hemicelulose & $\begin{array}{c}7,56 \pm \\
0,92\end{array}$ & $\begin{array}{c}19,01 \pm \\
1,26\end{array}$ & $\begin{array}{c}18,39 \pm \\
1,72\end{array}$ & $\begin{array}{c}11,13 \pm \\
0,61\end{array}$ & $20,55 \pm 1,22$ & $9,60 \pm 0,79$ & $\begin{array}{c}6,05 \pm \\
0,18\end{array}$ & $7,03 \pm 0,50$ & $\begin{array}{c}16,25 \pm \\
1,27\end{array}$ & $10,77 \pm 0,33$ & $\begin{array}{c}11,66 \pm 0,6 \\
8\end{array}$ \\
\hline Lignina & $\begin{array}{c}15,98 \pm \\
0,52\end{array}$ & $\begin{array}{c}19,72 \pm \\
1,05\end{array}$ & $\begin{array}{c}13,62 \pm \\
0,59\end{array}$ & $\begin{array}{c}41,65 \pm \\
2,29\end{array}$ & $9,77 \pm 0,67$ & $4,33 \pm 0,12$ & $\begin{array}{c}5,68 \pm \\
0,48\end{array}$ & $6,75 \pm 0,72$ & $\begin{array}{c}9,27 \pm \\
0,86\end{array}$ & $6,85 \pm 0,55$ & $0,59 \pm 0,04$ \\
\hline
\end{tabular}

AST: Açúcares solúveis totais; FDA: Fibra solúvel em detergente ácido; FDN: Fibra solúvel em detergente neutro; PB: Proteína Bruta 
Tabela 3 - Estimativa da produção de etanol em litros por tonelada de torta

\begin{tabular}{|l|c|c|c|c|}
\hline \multicolumn{1}{|c|}{ Biomassa } & $\begin{array}{c}\text { Fração amilácea } \\
\text { L ton. -1 }\end{array}$ & $\begin{array}{c}\text { Fração celulósica } \\
\text { L ton. -1 }\end{array}$ & $\begin{array}{c}\text { Fração hemicelulósica } \\
\text { L ton. }-1\end{array}$ & $\begin{array}{c}\text { Total } \\
\text { L ton. }-1\end{array}$ \\
\hline Nabo forrageiro & 18,2 & 46,8 & 35,8 & 100,8 \\
\hline Mamona & 37,2 & 81,5 & 39,2 & 157,9 \\
\hline Tremoço & 38,8 & 65,3 & 75,4 & 179,5 \\
\hline Pinhão-manso & 32,2 & 113,1 & 69,8 & 215,0 \\
\hline Crambe & 14,1 & 122,3 & 118,9 & 255,3 \\
\hline Girassol & 14,8 & 107,0 & 139,3 & 261,1 \\
\hline Algodão & 15,5 & 215,6 & 49,0 & 280,0 \\
\hline Macaúba & 149,7 & 74,2 & 62,0 & 285,9 \\
\hline Dendê & 32,4 & 196,2 & 71,9 & 300,5 \\
\hline Pequi & 175,4 & 107,6 & 105,0 & 388,0 \\
\hline Corda-de-viola & 56,2 & 216,1 & 123,1 & 395,3 \\
\hline
\end{tabular}

A figura 1 apresenta a estimativa de produção de etanol por hectare de plantio de cada oleaginosa estudada. Ganha destaque as culturas de macaúba e dendê, que possuem maior produtividade de etanol por hectare.

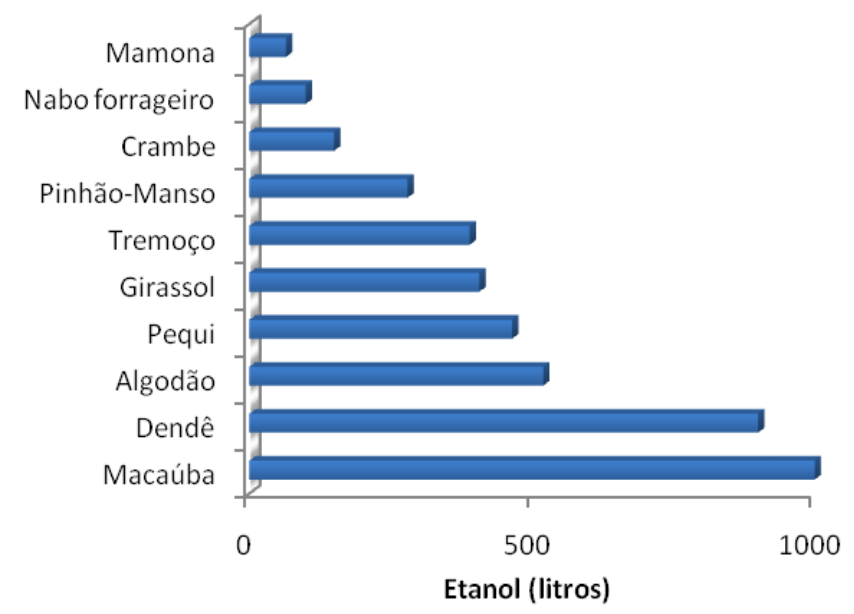

Figura 1: Estimativa dos valores de produtividade de etanol por hectare de plantio de mamona, nabo forrageiro, cambre, pequi, dendê, macaúba, girassol, pinhão-manso, algodão e tremoço com base nos percentuais de amido, celulose e hemicelulose presentes nas respectivas tortas.

A figura 2 apresenta valores estimados da produção de etanol por quantidade de biomassa gerada correspondente à extração de uma tonelada de óleo das oleaginosas e a demanda de etanol para a reação de transesterificação desse óleo. Apenas o nabo forrageiro e a mamona não atenderiam a totalidade da demanda, mas supririam boa parte do consumo de etanol. A torta de mamona é produzida na proporção de 1,2 toneladas por tonelada de óleo extraído (Tabela 1). Com a utilização da torta de mamona gerada pode-se obter até 180 litros 
de etanol. Já para o nabo forrageiro projeta-se uma produção de até 150 litros de etanol por tonelada de óleo extraído (Figura 2).

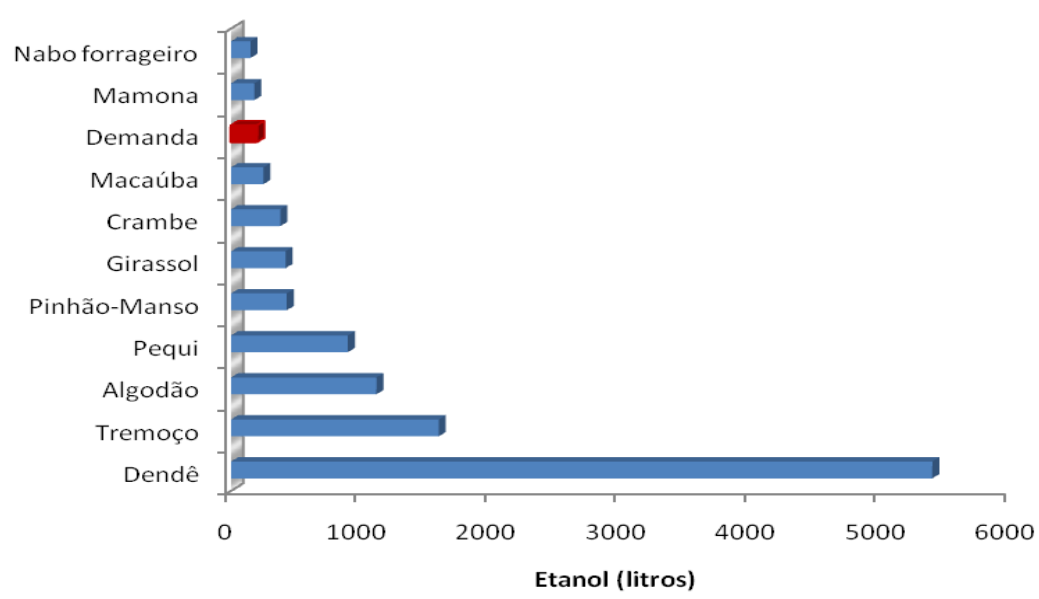

Figura 2: Valores estimados da produção de etanol por quantidade de biomassa gerada correspondente à extração de uma tonelada de óleo extraído das oleaginosas: mamona, nabo forrageiro, cambre, pequi, dendê, macaúba, girassol, pinhão-manso, algodão e tremoço. * Demanda de etanol para produção de biodiesel a partir de uma tonelada de óleo, via rota de transesterificação etílica.

Destaca-se a torta de dendê, com produção de etanol cerca de 30 vezes maior que a demanda. Com base nos valores de produtividade, 4,5 ton/ha, a geração de torta após extração do óleo pode chegar até 18 ton/ha, onde se projeta uma produção de até 5400 litros de etanol/ha (Figura 2).

A quantidade de torta de pinhão-manso gerada pela extração de uma tonelada de óleo (2,4 ton.) (Tabela 1) resultará produção de 430 litros de etanol. Este valor supera em dobro a demanda de etanol para produção de biodiesel por transesterificação (Figura 2). Valores semelhantes de produção de etanol também podem ser vistos para o girassol e para o cambre, apresentando uma produção de aproximadamente 490 e 380 litros de etanol (Figura 2), respectivamente.

A torta do coco da macaúba, além da projeção de produção de etanol de 286 litros provenientes das frações amilácea, celulósicas e hemicelulósicas (Tabela 2), pode-se estimar também uma produção de 74 litros de etanol provenientes da fração de açúcares livres presentes na torta, somando um total de 360 litros de etanol por tonelada de torta.

A torta de tremoço apresenta valores significativos de material lignocelulósico (Tabela 2). Considerando que certas espécies de tremoço apresentam cerca de $10 \%$ de óleo (Ribeiro, 2006), é possível estimar uma produção de 9 toneladas de torta por tonelada de óleo extraído, quantidade de biomassa que pode ser capaz de gerar até 1600 litros de etanol (Figura 2).

Segundo Macedo e colaboradores (2011) o teor de óleo encontrado na polpa de pequi representa cerca de $30 \%$. Considerando este teor lipídico, para cada tonelada de óleo de pequi é extraído são produzidos 2,3 toneladas de torta, sendo então projetada uma produção de quase 900 litros de etanol. 
Com base no teor de óleo das sementes de algodão, pode-se obter até 1120 litros de etanol para cada tonelada de óleo produzido (Figura 2), valores que superam 5 vezes a demanda de álcool para produção de biodiesel.

Ainda não há na literatura dados de produtividade da corda-de-viola, não podendo ser calculado a quantidade de torta produzida pela extração de uma tonelada de óleo. Sabe-se que pode ser produzidos cerca de 400 litros por tonelada dessa torta.

\section{CONCLUSÃO}

As projeções apresentadas neste estudo demonstraram que a produção de etanol a partir de tortas pode ser uma estratégia eficiente e promissora, com possibilidades reais de ser incorporada à cadeia produtiva do biodiesel, como forma de agregar valor a um coproduto abundante, além de contribuir com a redução dos custos de produção deste biocombustível.

\section{REFERÊNCIAS BIBLIOGRÁFICAS}

ASSOCIATION OF OFFICIAL ANALYTICAL CHEMISTRY - AOAC. Official methods of analysis of the Association of Official Analytical Chemistry. Washington: AOAC, 1992.

BERGMANN, J.C.; TUPINAMBA, D.D.; COSTA, O.Y.A.; ALMEIDA, J.R.M.; BARRETO, C.C.; BESSA, O. R.; NORONHA, H. M.; FERREIRA, F. R.; DOURADO, D. C.; CASTRO, R. P.; FARIA, BiodieselBr.com. Biodiesel representa 10,2\% da demanda mundial de óleos vegetais. Disponível em: http://www.biodieselbr.com/noticias/usinas/producao/biodieselrepresenta-10-2-da-demanda-mundial-de-oleos-vegetais.htm. Acesso em 20/04/2014.

BRASIL. MINISTÉRIO DE MINAS E ENERGIA. Análise de Conjuntura dos Biocombustíveis-2012. Empresa de Pesquisa e Energia, p. 33, 2013.

COIMBRA, M.C.; JORGE, N.. Proximate composition of guariroba (Syagrus oleracea), jerivá (Syagrus romanzoffiana) and macaúba (Acrocomia aculeata) palm fruits. F. Res. Int., v. 44, p. 2139-2142, 2011.

COMPANHIA NACIONAL DE ABASTECIMENTO - CONAB. Acompanhamento de safra brasileira: grãos. Oitavo levantamento, maio 2011 / Companhia Nacional de Abastecimento. Brasília: Conab, 2011.

CORZO-VALLADARES, P.A.; FERNÁNDEZ-MARTÍNEZ, J.M.; VELASCO, L.. Tocochromanol content and composition in Jatropha curcas seeds. In. Crops and Prod., v. 36, p. 304-307, 2012.

DAMBISKI, L.. Síntese de Biodiesel de Óleo de Nabo Forrageiro Empregando Metanol Supercrítico, 2007, Dissertação (Mestrado em Engenharia) - Programa de Pós-graduação em Engenharia Mecânica e de Materiais, Universidade Tecnológica Federal do Paraná, Curitiba, 2007.

DRUMOND, M.A.; SANTOS, C.A.F.; OLIVEIRA, V.R.; MARTINS, J.C.; ANJOS, J.B.; DUBOIS, M.; GILLES, K.A.; HAMILTON, K.K.; HEBERS, P.A.; SMITH, F..Colorimetric method for determination of sugar and related substances. Anal. Chem., v. 28, p. 356, 1956.

MACEDO, A. L., SANTOS, R. S., PANTOJA, L., PINTO, N. A. V. D.; SANTOS, A. S. Avaliação do aproveitamento do resíduo sólido da extração de óleo de mamona (Ricinus communis 1.) para produção de etanol. Rev. de Est. Un., v. 35, p. 75-85, 2009.

MARCHETTI, J.M.; MIGUEL, V.U.; ERRAZU A.F.. Possible methods for biodiesel production. Renew Sustain Energ Rev., v. 11, p. 1300, 2007. 
MASETTO, T.E.; QUADROS, J. B.;. MOREIRA, F.H.; RIBEIRO, D.M.; BENITES JUNIOR, I.;

MATH, M. C. K., S. P.; CHETTY, S. V.. Technologies for biodiesel production from used cooking oil: a review. Energ for Sustain Develop, v. 14, p.p. 339- 345, 2010.

MCCREADY, R.M.; GUGGOLZ, J.M.; SILVEIRA, V.; OWENS, H.S.. Determination of starch and amylose in veggetables. Aplication to pear. Anal Chem., v. 22, p 1156, 1950.

MISRA, R.D.K. Solar energy: recent advances and future outlook. Mat Tech, v. 28, p. 12, 2013.

RAMANJANEYULU, A.V.; VISHNUVARDHAN, A.; MADHAVI, A.. The impact of sowing date and irrigation regime on castor (Ricinus communis L.) seed yield, oil quality characteristics and fatty acid composition during post rainy season in South India. Ind Crops and Prod, v. 44, p. 25-31, 2013.

RIBEIRO, A.G.. Desenvolvimento de produto tipo shake utilizando farinha de tremoço doce (Lupinus albus) CV multolupa, decorticada e desengordurada. Araraquara: UNESP, 2006. Dissertação (Mestrado) - Programa de Pós Graduação em Alimentos e Nutrição, Faculdade de Ciências Farmacêuticas, Universidade Estadual Paulista. "Júlio de Mesquita Filho" UNESP, Araraquara, 2006.

SANTOS, E.A.. Caracterização de dendezeiros subespontâneos com base na produção de frutos e cachos. UESC, 2010. Dissertação (Mestrado) - Programa de Pós - graduação em Produção Vegetal, Universidade Estadual de Santa Cruz, 2010.

SILVA, J.S.O. Produtividade de óleo de palma na cultura do dendê na Amazônia oriental: influência do clima e material genético. UFV, 2006. Dissertação (Mestrado) - Programa de Pós Graduação em Fitotecnia, Universidade Federal de Viçosa - UFV, Viçosa, 2006.

TICKELL, J.. From the Fryer to the Fuel Tank: The Complete Guide to Using Vegetable 20 Oil as an Alternative Fuel. Tickell Energ Consult, p. 162, 2000.

VALLE, P.W.P.A. Produção de biodiesel via transesterificação do óleo de nabo forrageiro, 2009, Tese (Doutorado) - Departamento de Química do Instituto de Ciências Exatas, Universidade Federal de Minas Gerais - UFMG, 2009.

VAN SOEST, P. J. Development of a comprehensive system of feed analysis and its applications to forage. J. Anm. Sci., v. 26. p 119-128, 1967.

VAN SOEST, P.J.; MOORE, L.A. New chemical method for analysis of forages for the purpose of predicting nutritive value. Proc. IX inter. Grass. Gong. São Paulo. p 783-789, 1966.

VERA, R.; SOUZA, E.B.; FERNANDES, E.B.; NAVES, R.V.; SOARES JÚNIOR, M.S.; CALIARI, M.; XIMENES, P.A.. Caracterização física e química de frutos do pequizeiro (caryocar brasiliense camb.) oriundos de duas regiões no estado de Goiás, Brasil. Pesq Agropec Trop, v. 37, p. 93-99, 2007.

KOSARIC, N. AND VARDAR-SUKAN, F., Potential source of energy and chemical products. The biotechnology of ethanol: classical and future applications. Weinheim: Wiley VCH Verlag GmbH, p. 89-220, 2001. 